\title{
Resolution enhancement of transmission electron microscopy by super-resolution radial fluctuations
}

Cite as: Appl. Phys. Lett. 116, 044105 (2020); https://doi.org/10.1063/1.5128353

Submitted: 18 September 2019 . Accepted: 16 January 2020 . Published Online: 28 January 2020

Y. Zhang (D), S. Rouvimov, X. Yuan, K. Gonzalez-Serrano, A. C. Seabaugh (D), and S. S. Howard (i)
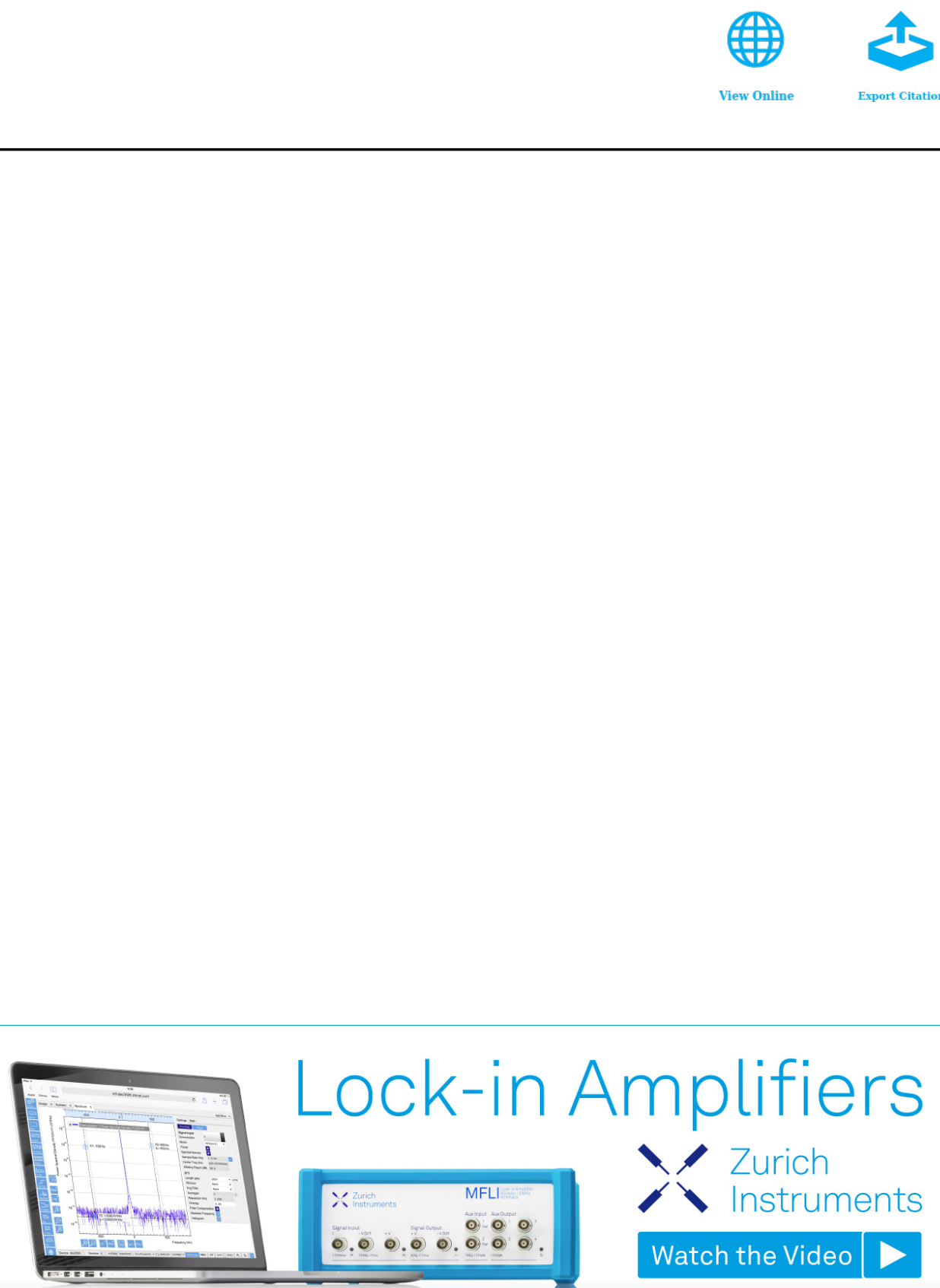


\title{
Resolution enhancement of transmission electron microscopy by super-resolution radial fluctuations
}

\author{
Cite as: Appl. Phys. Lett. 116, 044105 (2020); doi: 10.1063/1.5128353 \\ Submitted: 18 September 2019 . Accepted: 16 January 2020 . \\ Published Online: 28 January 2020
}
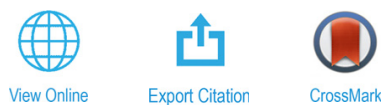

\author{
Y. Zhang, ${ }^{1,2}$ (D) S. Rouvimov, ${ }^{1,3}$ X. Yuan, ${ }^{1}$ K. Gonzalez-Serrano, ${ }^{7}$ A. C. Seabaugh, ${ }^{1, a)}$ (D) and S. S. Howard ${ }^{1, b)}$ (D) \\ AFFILIATIONS \\ 'Department of Electrical Engineering, University of Notre Dame, Notre Dame, Indiana 46556, USA \\ ${ }^{2}$ Caltech Optical Imaging Laboratory, Andrew and Peggy Cherng Department of Medical Engineering, California Institute \\ of Technology, Pasadena, California 91125, USA \\ ${ }^{3}$ Notre Dame Integrated Imaging Facility, University of Notre Dame, Notre Dame, Indiana 46556, USA \\ a) Electronic mail: aseabaug@nd.edu \\ ${ }^{b)}$ Electronic mail: showard@nd.edu
}

\begin{abstract}
Super-resolution fluorescence microscopy techniques have enabled dramatic development in modern biology due to their capability to discern features smaller than the diffraction limit of light. Recently, super-resolution radial fluctuations (SRRF), an analytical approach that is capable of generating super-resolution images easily without the need for specialized hardware or photoswitchable fluorophores, has been presented. While SRRF has only been demonstrated on fluorescence microscopes, in principle, this method can be used to generate superresolution images on any imaging platforms with intrinsic radial symmetric point spread functions. In this work, we show that SRRF can be utilized to enhance the resolution and quality of transmission electron microscopy (TEM) images. By including an image registration algorithm to correct for sample drift, the SRRF-TEM approach substantially enhances the resolution of TEM images of three different samples acquired with a commercial TEM system. We quantify the resolution improvement in SRRF-TEM and evaluate how SRRF parameters affect the resolution and quality of SRRF-TEM results.
\end{abstract}

Published under license by AIP Publishing. https://doi.org/10.1063/1.5128353

Fluorescence microscopy is essential in biomedical research as it allows researchers to observe specifically labeled cellular structures in their native physiological states. ${ }^{1}$ However, due to the diffraction of light, the resolution of fluorescence microscopy is limited to hundreds of nanometers. ${ }^{2}$ To discern features smaller than the diffraction limit in living cells, super-resolution microscopy techniques, notably stimulated emission depletion (STED) microscopy, ${ }^{3,4}$ photoactivated localization microscopy (PALM), ${ }^{5}$ stochastic optical reconstruction microscopy (STORM), ${ }^{6}$ structured illumination microscopy (SIM), and super-resolution optical fluctuation imaging (SOFI), ${ }^{9}$ have been demonstrated. Although these established super-resolution techniques have enabled dramatic development in modern biology, their implementations often require specialized, complicated optical setups or photoswitchable fluorophores, which are not accessible to many labs. Recently, super-resolution radial fluctuations (SRRF) has been demonstrated. ${ }^{10}$ Unlike the established super-resolution methods, SRRF does not require specialized hardware (e.g., depletion, structured illumination lasers, and high-speed cameras) or photoswitchable fluorophores; it can be implemented using almost any conventional fluorescence microscopes, such as widefield, confocal, and total internal reflection fluorescence (TIRF) microscopes using conventional, nonphotoswitchable fluorophores such as green fluorescent proteins (GFPs). ${ }^{10}$ With SRRF, super-resolution live-cell imaging over timescales ranging from minutes to hours can be achieved. ${ }^{1}$

Whereas super-resolution techniques were initially developed to overcome the diffraction limit of light in fluorescence microscopy, the methodologies behind these techniques can also be used to improve the resolution of other imaging modalities, such as transmission electron microscopy (TEM). Despite having subnanometer resolutions, ${ }^{12,13}$ many TEM images, especially those acquired from sensitive materials or captured with uncorrected aberrations, tend to have weak contrast, a low signal-to-noise ratio (SNR), and hence low resolution. ${ }^{14,15}$ Similar to fluorescence microscopy, when the resolution of TEM images becomes a limiting factor, a super-resolution approach becomes necessary. However, it is very difficult, if not impossible, to apply the established super-resolution microscopy techniques (STED, PALM, STORM, SIM, SOFI, etc.) in TEM, as these methods require either specialized hardware that processes photons, not electrons, or photoswitchable fluorophores, not atomic structures. Unlike these methods, SRRF utilizes the intrinsic radial symmetry of the 
microscope point spread function (PSF) and can be implemented easily without the requirement for additional hardware or photoswitchable emitters. Since TEM images also have intrinsic radial symmetric PSFs, ${ }^{16}$ it is feasible to utilize the SRRF approach to improve the resolution of TEM images.

In this work, we show that SRRF, a super-resolution approach developed for fluorescence microscopy, can be used to enhance the resolution and quality of TEM images. Like SRRF in fluorescence microscopy, SRRF-TEM requires a sequence of multiple TEM images acquired from the same field of view (FOV) of the sample. However, the repetitive imaging of the same structure in TEM results in significant sample drift caused by thermally induced structural transformation and transition, ${ }^{17,18}$ which should be compensated before SRRF processing. We demonstrate that a phase correlation-based image registration algorithm can correct for the sample drift in the TEM sequence. Three different samples were prepared and imaged using a commercial TEM system (FEI Titan 80-300). After the SRRF-TEM processing, substantial resolution enhancement can be observed on all the samples. We quantify the resolution improvement in SRRF-TEM results using Fourier ring correlation (FRC) resolutions. ${ }^{19}$ We also evaluate how different parameters in SRRF affect the resolution and quality of resulting SRRF-TEM images. We utilize multiple opensource ImageJ (US National Institutes of Health) plug-ins for SRRFTEM image processing, ${ }^{20}$ such that the SRRF-TEM approach can be easily accessed by the existing TEM users.

An overview of the SRRF-TEM approach and a block diagram summarizing the steps of SRRF-TEM are shown in Fig. 1, where the image registration step corrects for thermally induced sample drift, the spatial SRRF step enhances image resolution, and the temporal SRRF step suppresses shot noise-induced artifacts. First, a sequence consisting of multiple TEM images ( $N$ frames in total) is acquired from the same FOV of the sample. Whereas the number of frames $N$ is recommended to be as large as possible for SRRF in fluorescence microscopy, ${ }^{10}$ in TEM, due to the thermal effects induced by electron beams, it may be impossible to acquire $N>100$ frames of the same FOV without distorting the sample. As we demonstrate later in this work, a large number of total frames $(N>100)$ are not required to generate highquality SRRF-TEM images. In this work, we prepared samples from three different materials, i.e., polycrystalline gallium nitride $(\mathrm{GaN})$ on sapphire, polymer-coated carbon nanotubes (CNTs) on amorphized quartz, and nanoparticles (NPs). The TEM cross-sectional samples were prepared by Focus Ion Beam (FIB) using FEI Helios SEM/FIB dual beam equipment. This included depositing a protective iridium/ platinum cap over the polymer-coated CNTs to help preserve the structural integrity of the cross section. The samples were then imaged with a FEI Titan 80-300 electron microscope in high-resolution TEM (HRTEM) mode. ${ }^{21}$ To reduce thermally induced sample drift during the repetitive TEM acquisition, (a) each sample was imaged after waiting for an extended amount of time (e.g., $6 \mathrm{~h}$ ) and (b) a short exposure time was used for image acquisition. Consequently, the intensities and contrast of the raw TEM images were lower than typical.

Even though care has been taken to reduce the sample drift and the FEI Titan 80-300 has excellent stability with a total system drift lower than $0.3 \mathrm{~nm} / \mathrm{min}(\mathrm{rms}),{ }^{21}$ notable image drift was still observed from the raw TEM sequences. Two examples of such drift can be seen in Fig. 2. Unlike image drift in fluorescence microscopy, which is mostly caused by living sample movement or stage drift, ${ }^{22,23}$ TEM

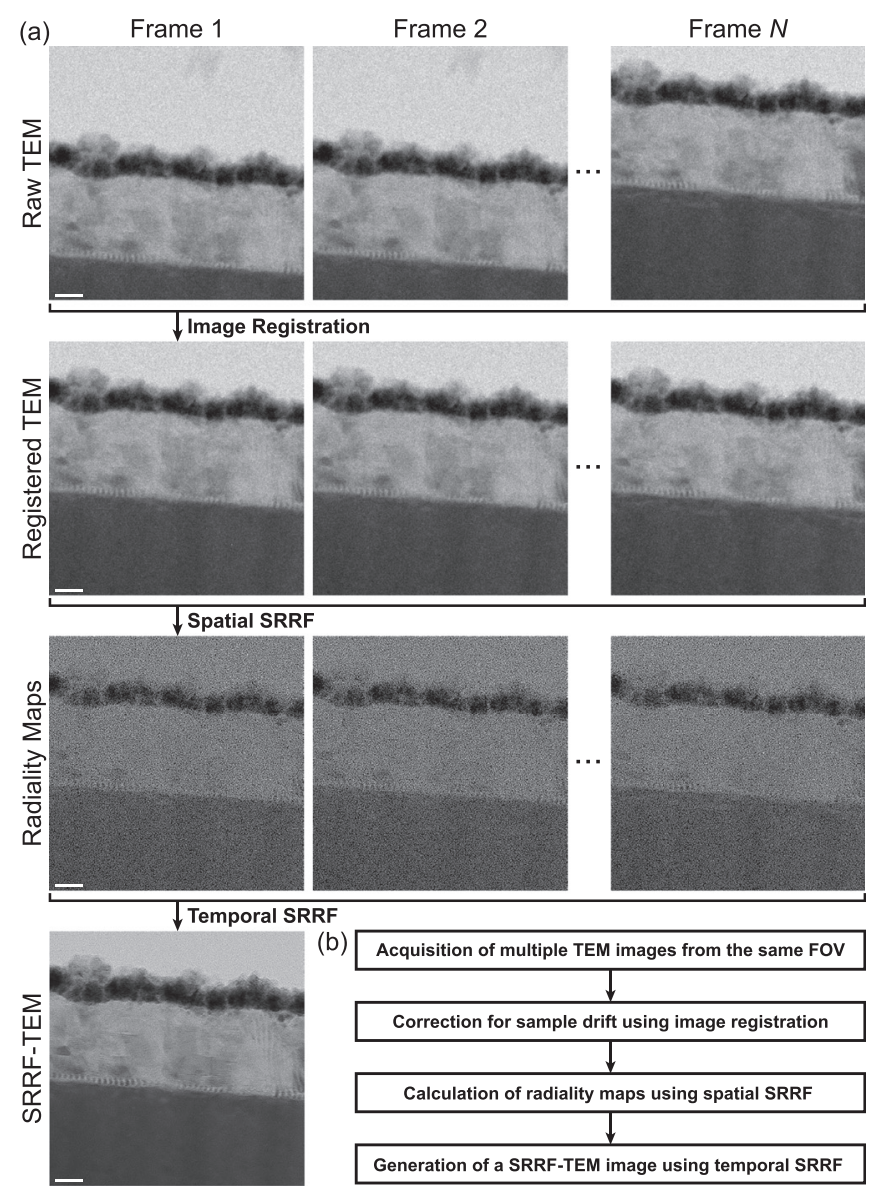

FIG. 1. Overview of the SRRF-TEM approach. (a) A sequence consisting of $N$ raw TEM images is acquired (shown here are the experimental TEM images of polycrystalline $\mathrm{GaN}$ on a sapphire sample). An image registration algorithm is used to correct for sample drift. Then, spatial and temporal SRRF algorithms are sequentially utilized to generate an SRRF-TEM image for the sequence. (b) Block diagram summarizing the steps of SRRF-TEM. Scale bar, $10 \mathrm{~nm}$.

image drift is caused by long-term thermal effects on samples and short-term fluctuations in the accelerating voltage and the objective lens current. ${ }^{17,24}$ Therefore, the second step of SRRF-TEM is to correct for the drift in the raw TEM sequence using image registration algorithms. Here, we employed the "Correct 3D drift" ImageJ plug-in to register the TEM sequence, where pair-wise phase correlation analysis was used to determine the translation between each TEM frame, and the calculated offsets were then applied to the sequence. ${ }^{25,26}$ Figure 2 shows the raw and registered sequences pseudo-color coded with each frame. Whereas the raw sequences are severely smeared, the image registration algorithm corrected for the drift such that no signs of drift can be observed in the registered sequences.

Next, the registered TEM sequence is processed using the SRRF algorithm, which consists of two distinct steps, a spatial and a temporal processing. ${ }^{10,11}$ The spatial SRRF step magnifies each raw pixel in a TEM image into subpixels (by default, 1 raw pixel is magnified and divided into $5 \times 5$ subpixels) and assigns a value termed "radiality" to each subpixel. The radiality value at a subpixel is obtained by 


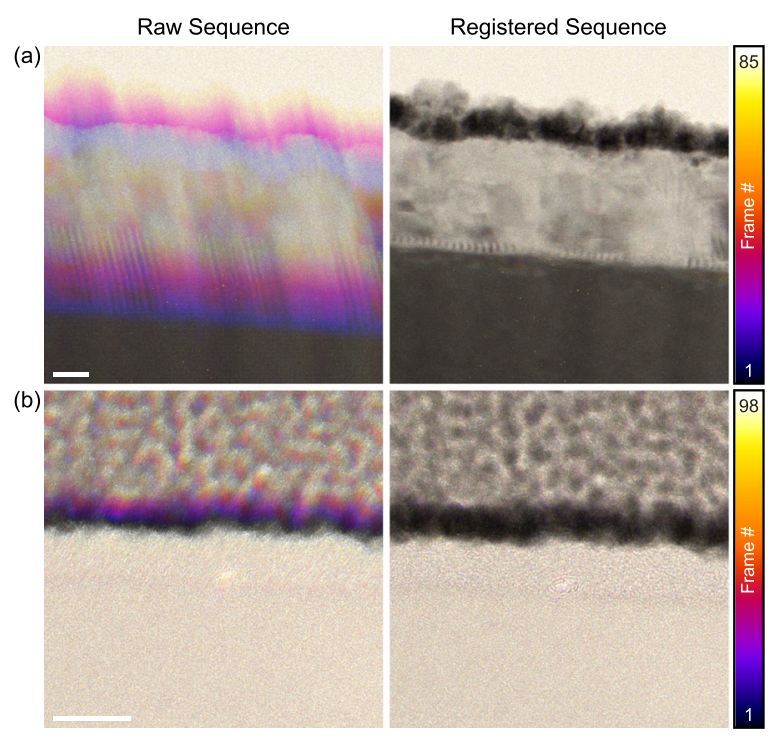

FIG. 2. Raw and registered experimental TEM sequences of (a) polycrystalline $\mathrm{GaN}$ on sapphire and (b) polymer-coated CNTs on amorphized quartz, pseudocolor coded to visualize the sample drift during the repeated image acquisition. The temporal scales for each frame in the sequences are shown on the right. Scale bar, $10 \mathrm{~nm}$.

calculating the degree of convergence of a group of intensity gradient vectors connecting the subpixel and a ring of nearby surrounding subpixels (by default, 12 gradient vectors are considered). The ring radius of these surrounding subpixels determines the precision of the radiality distribution and consequently, the resolution, or the full width at half maximum (FWHM) of the PSF, of the resulting SRRF-TEM images. The radiality value is related to the probability of the subpixel containing an emitter: for a subpixel located at or close to the center of an emitter, there will be a high degree of convergence of surrounding gradient vectors and hence a high radiality value; for a subpixel far away from an emitter, the degree of convergence, or the radiality, will be low. The radiality value can also be seen as a measurement of local radial symmetries within the image originated from the intrinsic radial symmetry of the microscope PSF: a high radiality value means that the gradients surrounding the subpixel have high radial symmetry. This is the theoretical basis that the SRRF method is applicable to both fluorescence microscopy and TEM images, as both images have intrinsic radial symmetric PSFs. ${ }^{16}$ The spatial SRRF processing is applied to each TEM image in the sequence, resulting in a sequence of the socalled radiality maps. Note that an intensity weighting procedure, which weights the radiality values by the raw pixel intensity, is used to conserve the quantitative information of raw images in radiality maps.

Finally, the sequence of radiality maps is subsequently processed by the temporal SRRF step to generate a single resolution-enhanced SRRF-TEM image. The temporal SRRF processing analyzes the fluctuations in radiality values within the sequence and extracts further information on the true positions of the emitters using cumulant analysis. Due to the shot noise induced by the discrete nature of electrons and the detection systems, ${ }^{27}$ some pixels in the raw images tend to have high intensity and hence high radiality values, even though they are not close to a true emitter; these pixels result in the grainy and noisy appearance of individual radiality maps shown in Fig. 1. To prevent these shot noise-induced artifacts from appearing in the resulting SRRF-TEM image, the temporal SRRF step analyzes the temporal statistics of the whole radiality map sequence such that the radiality fluctuations caused by noise are suppressed, while the radiality values of true emitters are preserved and possibly enhanced using high-order cumulant analysis (by default, first-order cumulant analysis, i.e., averaging, is used). We employed the "NanoJ-SRRF" ImageJ plug-in to perform both the spatial and temporal SRRF processing on the registered TEM sequence. ${ }^{10}$

Figure 3 shows the application of SRRF-TEM processing to TEM images of $\mathrm{Al}_{2} \mathrm{O}_{3}$ simulated using the QSTEM simulation software. ${ }^{28} \mathrm{~A}$ ground truth image of a $\mathrm{Al}_{2} \mathrm{O}_{3}$ structure $(20 \AA \times 20 \AA \times 20 \AA)$ was simulated using a high-resolution probe array $(400 \times 400$ pixels, $0.05 \AA$ resolution), while 64 raw TEM images of the same FOV were simulated using a low-resolution probe array $(50 \times 50$ pixels, $0.4 \AA$ resolution) with shot noise added (100 counts). The QSTEM simulation parameters were set as follows: $300 \mathrm{kV}$ high voltage, $-54.3 \mathrm{~nm}$ defocus, $1.0 \mathrm{~mm}$ spherical aberration, $1.0 \mathrm{~mm}$ chromatic aberration, $300 \mathrm{~K}$ temperature, $15 \mathrm{mrad}$ convergence angle, $5 \times 10^{8}$ brightness $\left(\mathrm{A} \mathrm{cm}^{-2} \mathrm{sr}^{-1}\right)$, and $1 \mu \mathrm{s}$ dwell time. The 64 raw TEM images were then used to generate the SRRF-TEM image shown in Fig. 3(a). As shown in Fig. 3(b), the SRRF-TEM image substantially enhances the resolution, contrast, and SNR of the raw TEM image. Compared to the ground truth, the SRRF-TEM image exhibits comparable image resolution and quality despite the lower contrast between the aluminum and oxygen atoms.

Figure 4 compares the raw TEM and SRRF-TEM images of crystalline and amorphous surfaces from three different samples. Four sequences consisting of $85,146,98$, and 83 raw TEM images were used to generate the SRRF-TEM results for the GaN, CNT, and NP samples in Fig. 4(a), respectively. We attempted to take more raw TEM images of the same FOV in our experiments; however, it turned out that doing so severely distorted and deformed the morphology of the sample, so we stopped the image acquisition and included only the undistorted frames in the SRRF-TEM analysis. As shown in Fig. 4(b), even with a limited number of frames $(N<100)$, a substantial enhancement in both image resolution and quality can be seen in SRRF-TEM images compared to their raw TEM counterparts: the blurry and low-contrast atomic layers and materials interfaces in the raw TEM images become cleaner, sharper, and with stronger contrast in the SRRF-TEM ones. Notably, the cross section of a CNT (Fig. 4, 3rd row), which can hardly be separated from its polymeric embedding layer in the raw TEM image, can be clearly discerned from the background in the SRRF-TEM one.
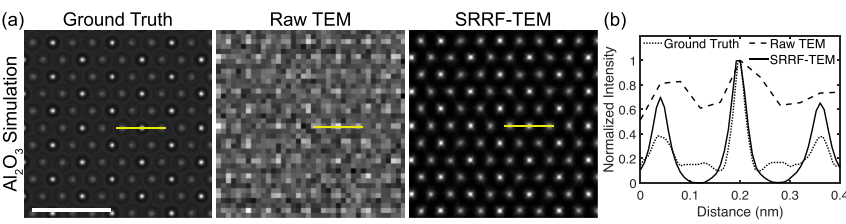

FIG. 3. Application of SRRF-TEM to simulated TEM images. (a) Ground truth (high-resolution, noise free) and raw TEM (low resolution, shot noise added) images of $\mathrm{Al}_{2} \mathrm{O}_{3}$ simulated using the QSTEM simulation software. The SRRF-TEM image was generated from 64 simulated raw TEM images. (b) Line profiles of the normalized pixel intensity values along the yellow lines in (a). Scale bar, $0.5 \mathrm{~nm}$. 


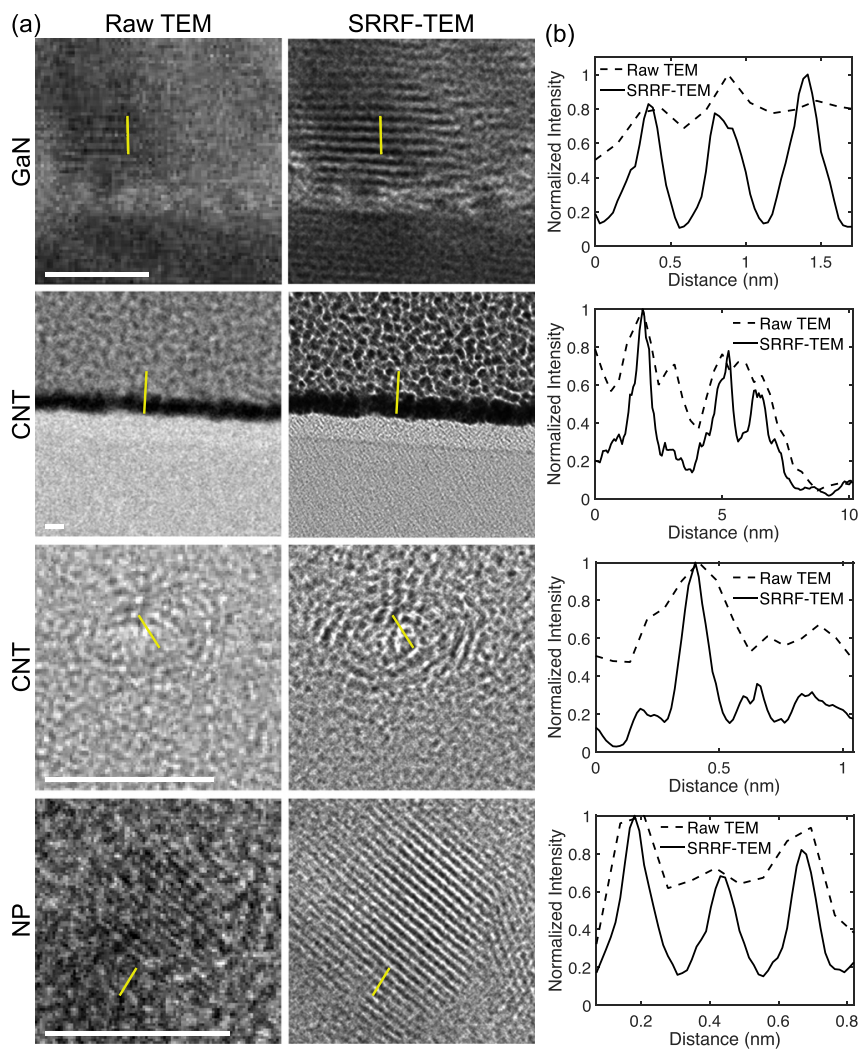

FIG. 4. Application of SRRF-TEM to experimental TEM images. (a) Comparison between raw TEM and SRRF-TEM images in four experiments acquired from three different samples: polycrystalline GaN on sapphire, polymer-coated CNTs on amorphized quartz, and nanoparticles (NPs). (b) Line profiles of the normalized pixel intensity values along the yellow lines in (a). Scale bar, $5 \mathrm{~nm}$.

To quantify the resolution enhancement of SRRF-TEM and evaluate how different parameters in the SRRF algorithm affect the results, we utilized Fourier ring correlation (FRC), an image-resolution measure that is based on the spectral SNR of the images and can be computed from the two images of the same scene (FOV), which differ only in noise content. ${ }^{19,29}$ Specifically, the raw TEM image sequences were evenly divided and processed into two SRRF-TEM images, which were used as two inputs for the "FRC" ImageJ plug-in to calculate the FRC resolution by locating the spatial frequency for which the FRC value dropped below a threshold (1/7). We first evaluated the effects of spatial SRRF parameters, i.e., the ring radius, on SRRF-FLIM results. The 98-frame raw TEM sequence of the CNT sample was divided into two independent 49 -frame sequences, which were processed through the SRRF-TEM approach multiple times using different ring radii; the two SRRF-TEM images after each processing were used to calculate the FRC resolution. This whole process was repeated for 16 experiments on different FOVs of the sample, and the results are summarized in Fig. 5. A relatively consistent resolution performance can be seen for a ring radius between 0.1 and 1.0; when the ring radius is larger than 1.0 , as the radius increases, the resolution becomes worse with larger means and standard deviations. This is consistent with the SRRF results acquired with fluorescence microscopes, where a smaller ring

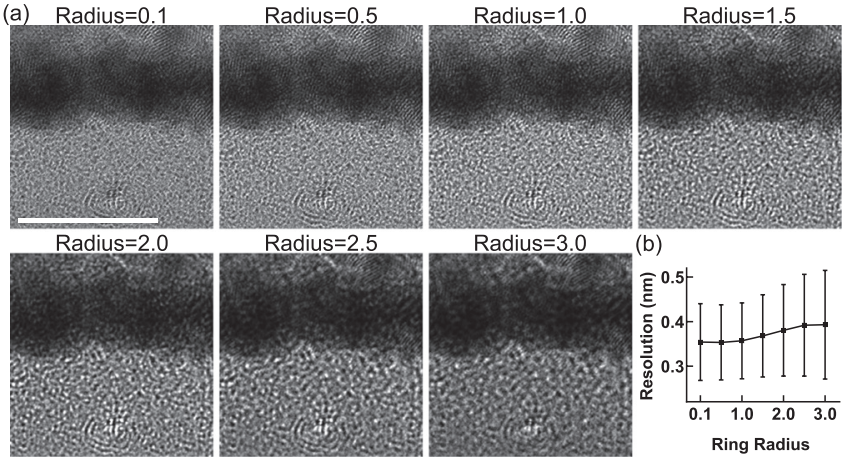

FIG. 5. Spatial SRRF parameters affect the resolution improvement in SRRF-TEM. (a) SRRF-TEM images generated from the same raw experimental TEM sequence of the CNT sample but using different ring radii. (b) FRC resolution as a function of the ring radius. Means and standard deviations are obtained from $n=16$ experiments. Scale bar, $10 \mathrm{~nm}$.

radius generally leads to a smaller FWHM of the PSF and consequently a higher resolution. ${ }^{10}$ Therefore, a ring radius between 0.1 and 1.0 is preferred for SRRF-TEM.

We used the same analysis to evaluate the effects of temporal SRRF parameters, including the total number of frames $(N)$ in the raw TEM sequence and the mode of cumulant analysis, on the resolution of SRRF-TEM results. To evaluate $N$, we chose the 146-frame raw TEMsequence of the CNT sample as it has sufficient frames to be divided into two sub-sequences with up to 64 frames. Figures 6(a) and 6(b) show that, as the number of frames increases, the resulting SRRF-TEM image appears less noisy, and both the mean and standard deviation of the FRC resolution becomes smaller. In other words, including more frames in the raw TEM sequence improves the quality and resolution of the SRRF-TEM result. However, as we mentioned earlier, for some sensitive samples, it may be impossible to acquire a large number of (e.g., $N>100$ ) raw TEM images without distorting it. Nevertheless, as Fig. 6(b) suggests, a sequence consisting of $N>30$ raw TEM frames can already provide a decent resolution-enhancement performance in SRRF-TEM. Finally, we divided the 146 -frame CNT sequence into two 72 -frame sub-sequences and evaluated the SRRF-TEM performance with respect to different modes of cumulant analysis, including temporal radiality maximum (TRM), temporal radiality average (TRA), temporal radiality pairwise product mean (TRPPM), and temporal radiality autocumulant (TRAC) of second, third, and fourth orders. The results are summarized in Figs. 6(c) and 6(d), where we also included a conventional average (AVG) projection of raw TEM images for comparison. Unlike SRRF in fluorescence microscopy, where higher-order cumulants (TRAC2-4) can provide higher resolution than lower-order ones (TRA, TRM, and TRPPM), ${ }^{10}$ SRRF-TEM performs best with lowerorder cumulants, especially TRPPM and the default TRA analysis.

In conclusion, we have demonstrated that SRRF, a superresolution approach developed for fluorescence microscopy, can be used to improve the resolution and quality of TEM images. Substantial enhancement of resolution has been demonstrated for SRRF-TEM images acquired from three different samples, where blurry and lowcontrast structural layers and interfaces become sharper and with stronger contrast. By quantifying and evaluating the SRRF-TEM performance using FRC resolutions, we have found a set of optimal 

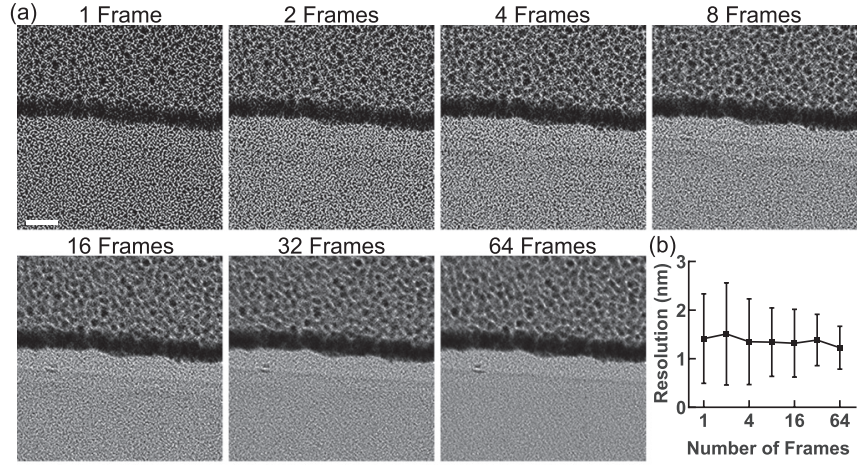

(c)

AVG

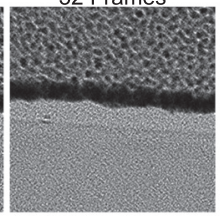

TRM
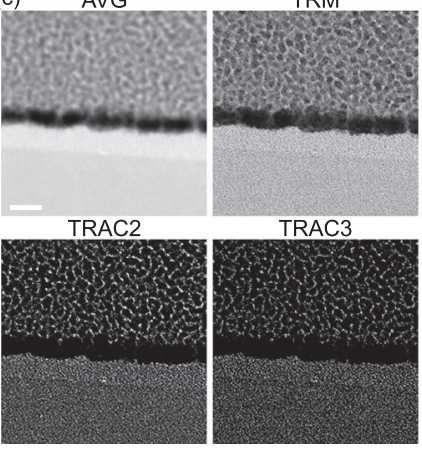

TRA

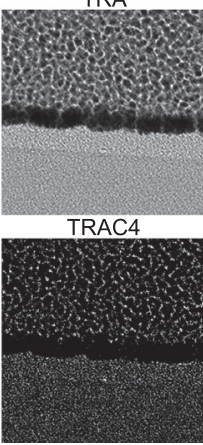

TRPPM

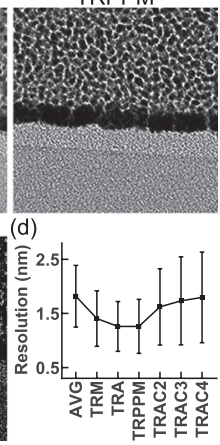

FIG. 6. Temporal SRRF parameters affect the resolution enhancement in SRRFTEM. (a) SRRF-TEM images generated from different numbers of frames within a raw experimental TEM sequence of the CNT sample. (b) FRC resolution as a function of the number of frames included in SRRF-TEM. (c) SRRF-TEM images generated from the same raw experimental TEM sequence of the CNT sample but using different modes of cumulant analysis. (d) Relationship between the FRC resolution and the cumulant analysis mode used in SRRF-TEM. Means and standard deviations in (b) and (d) are obtained from $n=16$ experiments. Scale bar, $10 \mathrm{~nm}$.

SRRF-TEM parameters (a ring radius between 0.1 and 1.0, a sequence of more than 30 raw TEM frames, and a cumulant analysis mode of TRA or TRPPM), which could be used as guidelines for interested TEM users. We believe that SRRF-TEM has the potential to become a powerful tool in materials research, and SRRF could also be used to enhance the resolution of other imaging modalities, such as photoacoustic imaging, magnetic resonance imaging, and neutron imaging.

The authors thank Greg Pitner and Philip Wong (Stanford University) for providing the CNTs imaged here. This material is based upon the work supported by the National Science Foundation under Grant No. CBET-1554516.

\section{REFERENCES}

'J. W. Lichtman and J.-A. Conchello, "Fluorescence microscopy," Nat. Methods 2, 910-919 (2005).

${ }^{2}$ S. W. Hell, "Far-field optical nanoscopy," Science 316, 1153-1158 (2007)

${ }^{3}$ S. W. Hell and J. Wichmann, "Breaking the diffraction resolution limit by stimulated emission: Stimulated-emission-depletion fluorescence microscopy," Opt. Lett. 19, 780-782 (1994).

${ }^{4}$ T. A. Klar, S. Jakobs, M. Dyba, A. Egner, and S. W. Hell, "Fluorescence microscopy with diffraction resolution barrier broken by stimulated emission," Proc. Natl. Acad. Sci. U. S. A. 97, 8206-8210 (2000).
${ }^{5}$ E. Betzig, G. H. Patterson, R. Sougrat, O. W. Lindwasser, S. Olenych, J. S. Bonifacino, M. W. Davidson, J. Lippincott-Schwartz, and H. F. Hess, "Imaging intracellular fluorescent proteins at nanometer resolution," Science 313, 1642-1645 (2006).

${ }^{6}$ M. J. Rust, M. Bates, and X. Zhuang, "Sub-diffraction-limit imaging by stochastic optical reconstruction microscopy (STORM)," Nat. Methods 3, 793-796 (2006).

${ }^{7}$ M. G. L. Gustafsson, "Surpassing the lateral resolution limit by a factor of two using structured illumination microscopy," J. Microsc. 198, 82-87 (2000).

${ }^{8}$ M. G. L. Gustafsson, "Nonlinear structured-illumination microscopy: Widefield fluorescence imaging with theoretically unlimited resolution," Proc. Natl. Acad. Sci. U. S. A. 102, 13081-13086 (2005).

${ }^{9}$ T. Dertinger, R. Colyer, G. Iyer, S. Weiss, and J. Enderlein, "Fast, backgroundfree, 3D super-resolution optical fluctuation imaging (SOFI)," Proc. Natl. Acad. Sci. U. S. A. 106, 22287-22292 (2009).

${ }^{10}$ N. Gustafsson, S. Culley, G. Ashdown, D. M. Owen, P. M. Pereira, and R. Henriques, "Fast live-cell conventional fluorophore nanoscopy with Image through super-resolution radial fluctuations," Nat. Commun. 7, 12471 (2016).

${ }^{11}$ S. Culley, K. L. Tosheva, P. M. Pereira, and R. Henriques, "SRRF: Universal livecell super-resolution microscopy,” Int. J. Biochem. Cell B. 101, 74-79 (2018).

${ }^{12}$ D. B. Williams and C. B. Carter, Transmission Electron Microscopy (Springer US, 1996).

${ }^{13}$ J. C. H. Spence, High-Resolution Electron Microscopy (Oxford University Press, 2013).

${ }^{14}$ M. Haider, S. Uhlemann, E. Schwan, H. Rose, B. Kabius, and K. Urban, "Electron microscopy image enhanced," Nature 392, 768-769 (1998).

${ }^{15}$ P. E. Batson, N. Dellby, and O. L. Krivanek, "Sub-ångstrom resolution using aberration corrected electron optics," Nature 418, 617-620 (2002).

${ }^{16}$ A. R. Lupini and N. de Jonge, "The three-dimensional point spread function of aberration-corrected scanning transmission electron microscopy," Microsc. Microanal. 17, 817-826 (2011).

${ }^{17}$ Z. Wang, "New developments in transmission electron microscopy for nanotechnology," Adv. Mater. 15, 1497-1514 (2003).

${ }^{18} \mathrm{X}$. Sang and J. M. LeBeau, "Revolving scanning transmission electron microscopy: Correcting sample drift distortion without prior knowledge," Ultramicroscopy 138, 28-35 (2014).

${ }^{19}$ R. P. J. Nieuwenhuizen, K. A. Lidke, M. Bates, D. L. Puig, D. Grünwald, S. Stallinga, and B. Rieger, "Measuring image resolution in optical nanoscopy," Nat. Methods 10, 557-562 (2013).

${ }^{20}$ C. A. Schneider, W. S. Rasband, and K. W. Eliceiri, "NIH Image to ImageJ: 25 years of image analysis," Nat. Methods 9, 671-675 (2012).

${ }^{21}$ A. Thust, J. Barthel, and K. Tillmann, "FEI Titan 80-300 TEM," J. Large-Scale Res. Facil. 2, A41 (2016).

${ }^{22}$ M. J. Mlodzianoski, J. M. Schreiner, S. P. Callahan, K. Smolková, A. Dlasková, J. Šantorová, P. Ježek, and J. Bewersdorf, "Sample drift correction in 3D fluorescence photoactivation localization microscopy," Opt. Express 19, 15009 (2011).

${ }^{23}$ S. H. Lee, M. Baday, M. Tjioe, P. D. Simonson, R. Zhang, E. Cai, and P. R. Selvin, "Using fixed fiduciary markers for stage drift correction," Opt. Express 20, 12177 (2012).

${ }^{24} \mathrm{~J}$. Barthel and A. Thust, "Aberration measurement in HRTEM: Implementation and diagnostic use of numerical procedures for the highly precise recognition of diffractogram patterns," Ultramicroscopy 111, 27-46 (2010).

${ }^{25}$ S. Preibisch, S. Saalfeld, and P. Tomancak, "Globally optimal stitching of tiled 3D microscopic image acquisitions," Bioinformatics 25, 1463-1465 (2009).

${ }^{26}$ A. Parslow, A. Cardona, and R. J. Bryson-Richardson, "Sample drift correction following 4D confocal time-lapse imaging," J. Vis. Exp. 86, e51086 (2014).

${ }^{27}$ T. Seki, Y. Ikuhara, and N. Shibata, "Theoretical framework of statistical noise in scanning transmission electron microscopy," Ultramicroscopy 193, 118-125 (2018).

${ }^{28}$ C. Koch, Determination of Core Structure Periodicity and Point Defect Density Along Dislocations (Arizona State University, 2002).

${ }^{29}$ M. Unser, B. L. Trus, and A. C. Steven, "A new resolution criterion based on spectral signal-to-noise ratios,” Ultramicroscopy 23, 39-51 (1987). 